\title{
The spectrum of underlying diseases in children with autoimmune hemolytic anemia
}

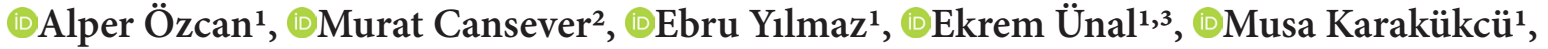 \\ (D)Türkan Patıroğlu ${ }^{1,2}$ \\ ${ }^{1}$ Erciyes University, Faculty of Medicine, Department of Pediatrics, Division of Pediatric Hematology Oncology \& Pediatric HSCT Unit, \\ Kayseri, Turkey \\ ${ }^{2}$ Erciyes University, Faculty of Medicine, Department of Pediatrics, Division of Pediatric Immunology, Kayseri, Turkey \\ ${ }^{3}$ Erciyes University, Molecular Biology and Genetic Department, Gevher Nesibe Genom and Stem Cell Institution, Genome and Stem Cell \\ Center (GENKOK), Kayseri, Turkey
}

Cite this article as: Özcan Ai Cansever M, Yılmaz E, Ünal E, Karakükcü M, patıroğlu T. The spectrum of underlying diseases in children with autoimmune hemolytic anemia. J Health Sci Med 2021; 4(6): 772-778.

\begin{abstract}
Aim: Autoimmune hemolytic anemia (AIHA) is characterized by the production of antibodies directed against red blood cells. We aimed to describe the clinical presentation, hematologic and biochemical profiles, treatment modalities, underlying diseases and outcomes in children suffering from AIHA.

Material and Method: In a retrospectively manner, we consecutively recrutied 62 children (aged 1 month-18 years) with AIHA who had been followed in Erciyes University Child Hospital between January, 2000 and November, 2017.

Results: The mean age at time of diagnosis was $61.9 \pm 51.9$ months (range:1-192) in 62 children including 28 girls and 34 boys. The most common complaints included fatigue and fever while the most common findings were jaundice and hepatosplenomegaly. In 22 children (35.4\%), there was a comorbid, active, non-specific infection (upper respiratory tract infection, lower respiratory tract infection, diarrhea, urinary tract infection). At time of diagnosis, mean hemoglobin $(\mathrm{Hb}$ ) level was $6.9 \pm 2.5 \mathrm{~g} / \mathrm{dL}$ (range: 5-9). The glucocorticoid therapy was given in majority of the patients while no medical treatment was given to 4 patients. Of the patients with primary AIHA, 6 patients were unresponsive to the treatment while one patient responded partially. It was found that there were underlying risk factors in 36 patients with secondary AIHA, as immunodeficiency and autoimmune disorders being the most common risk factors.

Conclusion: The immunodeficiencies were highly prevalent in children included. By advance of whole exome sequencing technology, we believe that primary immunodeficiencies was the most common underlying disease in our study detected quite high in presented children.
\end{abstract}

Keywords: Autoimmune hemolytic anemia, children, immunodeficiency

\section{INTRODUCTION}

Autoimmune hemolytic anemia (AIHA) is characterized by the production of antibodies directed against red blood cells, in which the red cell lysis occurs either by the mononuclear phagocytic system or by the complement system. The AIHA prevalence is still unknown at childhood, but it likely increases by advancing age as for most autoimmune disorders. In some studies, the AIHA prevalence was estimated as $0.2-0.8$ per in 100,000 person-years $(1,2)$.

In infantile period, it mostly develops due to viral and bacterial infections or vaccination. However, in teenagers and young adults, there is an increased association with an underlying systemic illnesses, most commonly with immunodeficiency, malignancy and autoimmune disorders (systemic lupus erythematosus (SLE), collagen vascular diseases) (3).

The AIHA diagnosis generally depends on clinical features, positive direct anti-globulin test (DAT) / Coombs test, laboratory analysis for hemolysis,anemia, hyperbilirubinemia, reticulocytosis. (4).

In hemodynamically stable patients with $\mathrm{Hb}$ level $<7 \mathrm{~g} /$ $\mathrm{dL}$, the erythrocyte transfusion is based on the American Association of Blood Banks (AABB) guidelines. If an AIHA patient has severe anemia and cardiopulmonary symptoms due to anemia,erythrocyte transfusion is required (5). 
In general, glucocorticoids are first-line treatment options due to their immunosuppressive effect. The additional treatment options may include intravenous immunoglobulin (IVIG) therapy, splenectomy, danazol, or immunosuppressive agents (6).

In this study, we aimed to describe the clinical presentation,hematologic and biochemical profiles, treatment options, underlying diseases and outcomes in children suffering from AIHA in Kayseri, Central Anatolia, Turkey

\section{MATERIAL AND METHOD}

We retrospectively reviewed children (aged 1 months-18 years) who were diagnosed as AIHA at Pediatric Hematology and Oncology Department of Erciyes University, Medicine School between January 2000 and November 2017. From medical records and outpatient clinic database, we initially searched data regarding history and physical examination, first-line tests including hemogram, White blood cell (WBC), peripheral smear for red cell morphology for hemolysis, reticulocyte count and reticulocyte smear, biochemical hemolysis parameters (DAT, haptoglobin, lactate dehydrogenase (LDH), indirect bilirubin), blood typing, hepatic and renal function tests, and urinalysis. In addition, we also searched data regarding second-line tests including viral markers (hepatitis B, C and HIV, EBV, CMV, Parvovirus B19, HSV serology), immune-hematological parameters (C3, C4, CH50, autoimmune markers including thyroid auto-antibodies, immunoglobulin class, lymphocyte subpopulation, double-negative T cells, immune disease panel) in patients diagnosed AIHA.

The patients with clinical findings and laboratory results of AIHA such as anemia (including elevated reticulocyte count and blood smear with hemolysis), positive DAT, low haptoglobin levels, increased indirect bilirubin and $\mathrm{LDH}$ levels were reviewed. For all records reviewed, the study was carried out with the permission of Erciyes University Faculty of Medicine Clinical Researchs Ethics Committee (Date: 16.06.2017, Decision No: 2017/348). All procedures were carried out in accordance with the ethical rules and the principles of the Declaration of Helsinki.

The AIHA classification was made based on different serological antibodies (IgG, IgM, IgA, C3, and/or combinations) for different serological types, disease severity and treatment outcome. Patients diagnosed with AIHA were classified as primary and secondary AIHA according to associated and potentially causative disorders including infections, autoimmune disorders, and malignancies. Whole exome sequencing (WES) was performed in the patients diagnosed with AIHA, in whom etiology could not be determined.
The children aged 1 month $\leq$ or $\geq 18$ years were excluded from the study because of unique features. The patients with transfusion-related AIHA, during bone marrow transplantation or those congenital hemolytic disorders were also excluded.

For treatment response, the complete response was defined as achieving $\mathrm{Hb}$ concentration $\geq$ lower limit of normal for age with no signs of hemolysis, i.e. normal reticulocyte count and bilirubin concentration while the partial response was defined as an increase in $\mathrm{Hb}$ level by $\geq 2 \mathrm{~g} / \mathrm{dLb}$ t not reaching a normal value for age. No response was defined as an increase in $\mathrm{Hb}$ level by<2 g/ $\mathrm{dL}$ and/or dependence on transfusions (7).

\section{Statistical Analysis}

To summarize data, descriptive statistics were given in tables as mean \pm standard deviation or median and interquartile range for continuous variables according to the distribution. The categorical variables were summarized as counts and percent. In comparisons between categorical variables, if the values observed in $2 \times 2$ tables are 5 and above, Pearson Chi-Square was used while Fisher Exact test was used cases where it is below 5. The RxC tables were used in Fisher's Freeman Halton Test. The statistical analyses were performed using Jamovi project software [(2018, Version 0.9.2.8), (Computer Software), (Retrieved from https://www.jamovi.org)]. The significance level was set as 0.05 ( $\mathrm{p}$ value).

\section{RESULTS}

The study included 62 patients who were diagnosed as AIHA between 2000 and 2017. Of the patients included, $34(54.8 \%)$ were boys and $28(45.2 \%)$ were girls. The mean age at time of diagnosis was $61.9 \pm 51.9$ months (range: 1-192).

There was comorbid, active, non-specific infection (upper respiratory tract infection, lower respiratory tract infection, diarrhea, urinary tract infection) in 22 patients (35.4\%). Table 1 summarizes clinical features of the patients. The patients were classified into two groups as primary and secondary AIHA according to the etiologic causes. When groups were compared regarding age, gender, blood groups, complaints on admission, physical examination findings, relapsefrequency and frequency of accompanying diseases, no significant difference was found.

When the complete blood counts and biochemical parameters were compared between the patients with primary and secondary hemolytic anemia, no significant difference was found inWBC, Hb level, platelet count, reticulocyte percentage, lymphocyte count, eosinophil count, neutrophil/lymphocyte rate, haptoglobin level, 


\begin{tabular}{|c|c|}
\hline Age, month (Range: 1-192 months) & $61.9 \pm 51.9$ \\
\hline \multicolumn{2}{|l|}{ Gender } \\
\hline Male & $34(54.8 \%)$ \\
\hline Female & $28(45.2 \%)$ \\
\hline \multicolumn{2}{|l|}{ AIHA } \\
\hline Primary & $26(41.9 \%)$ \\
\hline Secondary & $36(49.1 \%)$ \\
\hline \multicolumn{2}{|l|}{ Complaints } \\
\hline Fatigue & $12(19.4)$ \\
\hline Fever & $5(8.1)$ \\
\hline Concentrated urine & $4(6.5)$ \\
\hline Uneasiness & $3(4.8)$ \\
\hline Loss of appetite & $2(3.2)$ \\
\hline Diarrhea & $1(1.6)$ \\
\hline Vomiting & $1(1.6)$ \\
\hline Pain & $1(1.6)$ \\
\hline Convulsion & $1(1.6)$ \\
\hline \multicolumn{2}{|l|}{ Physical examination findings } \\
\hline Icterus & $34(54.8)$ \\
\hline Hepatosplenomegaly & $33(53.2)$ \\
\hline Paleness & $31(50)$ \\
\hline Murmur & $17(27.4)$ \\
\hline Tachycardia & $7(11.3)$ \\
\hline Splenomegaly & $6(9.7)$ \\
\hline Hepatomegaly & $3(4.8)$ \\
\hline Microcephaly & $1(1.6)$ \\
\hline Oral moniliasis & $1(1.6)$ \\
\hline \multicolumn{2}{|l|}{ Laboratory results of AIHA patients } \\
\hline $\mathrm{WBC}\left(10^{3} / \mu \mathrm{L}\right)$ & $11855.8 \pm 7379.5$ \\
\hline $\operatorname{Hgb}(g / d L)$ & $6.9 \pm 2.5$ \\
\hline $\operatorname{PLT}\left(10^{3} / \mu \mathrm{L}\right)$ & $335400 \pm 186080.7$ \\
\hline \% RTC & $10.8 \pm 7.5$ \\
\hline $\operatorname{NEU}\left(10^{3} / \mu \mathrm{L}\right)$ & $5972.1 \pm 5534.1$ \\
\hline $\operatorname{LYMP}\left(10^{3} / \mu \mathrm{L}\right)$ & $3802.9 \pm 2634$ \\
\hline $\operatorname{EOS}\left(10^{3} / \mu \mathrm{L}\right)$ & $176.1 \pm 186.4$ \\
\hline NEU/LYMPH (\%) & $2 \pm 1.8$ \\
\hline Monocyte $\left(10^{3} / \mathrm{L}\right)$ & $598.2 \pm 601.1$ \\
\hline Total bilirubin (mg/dL) & $4.4 \pm 4.4$ \\
\hline Free bilirubin(mg/dL) & $0.8 \pm 1.3$ \\
\hline Haptoglobulin (g/L) & $16.9 \pm 36.9$ \\
\hline $\mathrm{LDH}(\mathrm{u} / \mathrm{L})$ & $698.3 \pm 872.1$ \\
\hline
\end{tabular}

and LDH level. When the neutrophil counts were compared between groups, it was found that neutrophil count was significantly lower in patients with secondary AIHA than in patients with primary AIHA $(\mathrm{p}<0.05)$. It was found that total and free bilirubin levels were higher in patients with primary AIHA compared to those with secondary AIHA $(\mathrm{p}<0.05)$.

DAT was performed in all the patients for the presence of antibody. A significant difference was found between DAT positivity and $\mathrm{Hb}$ level in patients with both primary and secondary AIHA. When the distribution of antibody types was evaluated in 32 cases (51.6\%), the DAT was positive for IgG and negative for C3d,while in 19 cases (30.7\%) both were positive. Distributions of AIHA groups, DAT positivity scores, mean treatment durations and response conditions to treatment according to $\mathrm{Hb}$ levels were given in Table 2.

As the first-line treatment, 12 patients (19.4\%) received IVIG therapy while 31 patients (50\%) received high-dose steroid treatment $(10-30 \mathrm{mg} / \mathrm{kg} /$ day $)$, and 15 patients (24.2\%) received low-dose steroid treatment $(2 \mathrm{mg} / \mathrm{kg} /$ day). No medical treatment was given to 4 patients (6.4\%). The patients unresponsive to IVIG were given high-dose steroid treatment $(10-30 \mathrm{mg} / \mathrm{kg} /$ day $)$ or low-dose steroid treatment $(2 \mathrm{mg} / \mathrm{kg} /$ day $)$. Only one patient achieved remission with IVIG treatment. Regarding treatment response, there was no significant difference between the two groups received low-dose steroid treatment $(2 \mathrm{mg} / \mathrm{kg} /$ day) and high-dose steroid treatment $(10-30 \mathrm{mg} / \mathrm{kg} /$ day $)$. Eleven patients (17.7\%) did not respond to the treatment. One of the patients died. The relapse was seen in 8 patients (29.6\%) in the group received IVIG and/or low-dose steroid $(2 \mathrm{mg} / \mathrm{kg} /$ day) whereas in 5 patients $(16.1 \%)$ in the group received high dose steroid (10-30 mg/kg/day). There was no significant difference in relapse between groups. The combined treatments were often used in treatment. Apart

\begin{tabular}{|c|c|c|c|c|c|}
\hline & \multicolumn{4}{|c|}{ Hemoglobin level (gr/dL) } & \multirow[t]{2}{*}{$\mathbf{p}$} \\
\hline & $0-4$ & 4-8 & 8-12 & Total & \\
\hline AIHA & & & & & $0.166^{*}$ \\
\hline Primary & $1(2.9)$ & $24(70.6)$ & $9(26.5)$ & 34 & \\
\hline Secondary & $4(14.3)$ & $14(50)$ & $10(35.7)$ & 28 & \\
\hline DAT & & & & & $0.028^{*}$ \\
\hline+ & $0(0)$ & $1(50)$ & $1(50)$ & 2 & \\
\hline++ & $2(12.5)$ & $5(31.3)$ & $9(56.3)$ & 16 & \\
\hline+++ & $3(13)$ & $16(69.6)$ & $4(17.4)$ & 23 & \\
\hline++++ & $0(0)$ & $16(76.2)$ & $5(23.8)$ & 21 & \\
\hline Duration of treatment (day) & $180(30-180)$ & $142.5(90-280)$ & $40(10-75)$ & - & $<0.001^{* *}$ \\
\hline Response to treatment & & & & & $0.104^{\star}$ \\
\hline $\mathrm{CR}$ & $4(8.3)$ & $29(60.4)$ & $15(31.3)$ & 48 & \\
\hline NR & $1(9.1)$ & $9(81.8)$ & $1(9.1)$ & 11 & \\
\hline PR & $0(0)$ & $0(0)$ & $1(100)$ & 1 & \\
\hline No treatment & $0(0)$ & $0(0)$ & $2(100)$ & 2 & \\
\hline
\end{tabular}


from corticosteroid and IVIG treatments, 7 patients who were refractory or not responding to the treatment or with relapse received cyclosporine treatment while 3 patients received mycophenolate mofetil (MMF) treatment, 2 patients received rituximab (RTX), 3 patients underwent plasma exchange and 4 patients underwent splenectomy. No relapse was observedin patients who received RTX and plasmapheresis. Relapses were seen all 3 patients who underwent splenectomy.The duration of treatment in patients underwent RTX and plasmapheresis was longer than those received steroid or IVIG treatment as first-line treatment.

However, in group with DAT ++ results, the most patients had $\mathrm{Hb}$ levels between $8-12 \mathrm{mg} / \mathrm{dL}$ (high), indicating a significant difference $(\mathrm{p}=0.028)$. When duration of treatment was evaluated, it was significantly shorter in the group with high level of $\mathrm{Hb}$ compared to the other groups $(p<0.001)$, which was an expected condition as high severity of anemia would cause low levels of $\mathrm{Hb}$ and require longer duration of treatment. There was no significant difference in treatment response according to Hb levels.

The mean number of erythrocyte transfusions was $3.4 \pm 6.2$ in the group with primary AIHA while it was $2 \pm 1.8$ in the group with secondary AIHA, indication to significant difference. Of the patients with primary AIHA, 6 (54.5\%) were unresponsive to the treatment, and one patient responded partially. Of the patients with secondary AIHA, 5 (45.5\%) were unresponsive to the treatment. There was no significant difference in treatment response between the groups with primary and secondary AIHA .

The rate of drug-related side effects was $44.4 \%$ in low-dose steroid group and $32.3 \%$ in high-dose steroid group, indicating no significant difference between groups. The most common side effects were gastrointestinal complaints, increased appetite, stria formation, hypertrichosis, Cushingoid appearance, and thrombosis. In our study, one patient had cortical venous sinus thrombosis induced by steroid treatment which was previously reported (8).

When the underlying diseases were assessed in patients who were treated for secondary AIHA, it was found that there was immunodeficiency in 14 (38.9\%), Evans syndrome in $6(16.6 \%)$, Hodgkin's lymphoma in 3 (8.3\%), SLE in $4(11.1 \%)$, diabetes mellitus (DM) in one, autoimmune hepatitis in one and infection secondary AIHA in 4 patients. Table 3 presents immunodeficiencies detected in patients who were followed up due to secondary AIHA.

Relapse was seen in 13 patients (21.0\%) while 29 patients $(46.8 \%)$ had a disease that had already been followed up.
Table 3. Diseases found in patients diagnosed with secondary AIHA

\begin{tabular}{|lc|}
\hline & n (\%) \\
\hline PrimaryAIHA & $26(41.9)$ \\
Secondary AIHA & $36(49.1)$ \\
Autoimmune Diseases & \\
Evans'syndrome & $6(9.7)$ \\
SLE & $4(6.5)$ \\
Autoimmune hepatitis & $1(1.6)$ \\
Type-1 Diabetes mellitus & $1(1.6)$ \\
Hashimoto's thyroiditis & $1(1.6)$ \\
Autoimmune neuropathy & $1(1.6)$ \\
Immunodeficiencies & \\
SCID (undefined) & $3(4.8)$ \\
SCID (defined) & $7(11.2)$ \\
$\quad$ PGM3 mutation & $2(3.2)$ \\
LRBA deficiency & $2(3.2)$ \\
RAG 1 deficiency & $1(1.6)$ \\
XAP 70 deficiency & $1(1.6)$ \\
CVID 2 deficiency & $1(1.6)$ \\
ALPS & $2(3.2)$ \\
Malignancies & $2(3.2)$ \\
Hodgkin's lymphoma & $3(4.8)$ \\
Infection & $1(1.6)$ \\
HBV & $1(1.6)$ \\
HEV & $1(1.6)$ \\
Varicella & $1(1.6)$ \\
\hline Tuberculosis & \\
\hline CVID: Common variable immunodeficiency,SCID:Severe combined \\
immunodeficiency
\end{tabular}

\section{DISCUSSION}

The AIHA causes erythrocyte destruction as a result of autoantibodies binding to erythrocyte surface membrane. Its incidence is higher than that of aplastic anemia. The primary AIHA is seen in young children. Although mean age varies according to the regions in recent studies, it was reported as 5.3 years in a study performed in Turkey, 11 years in a study in India, 3.8 years in a study in France and 10 years in a study by Mayo Clinic (USA) (9-12).

A mild fever may be seen in children. Paleness and scleral icterus are commonly seen in patients. Anemia-induced systolic murmur, tachycardia and hepatosplenomegaly are commonly seen physical examination findings. In agreement with pediatric series in literature, the most common complaints on admission were fatigue, fever and concentrated urine while paleness, jaundice and hepatosplenomegaly were the most common physical examination findings in our patients (11). Apart from expected clinical presentations, we also had patients who presented with life-threatening heart failure caused by severe anemia. Life-threatening hemolytic complications may be seen during erythrocyte transfusions to the patients with AIHA $(13,14)$. We generally had crossmatching problems in our patients requiring erythrocyte transfusion and no serious hemolytic reaction transfusion was observed. 
In children, the warm AIHA is generally provoked by viral infections and hemolysis may occur. In the literature, warm AIHA cases caused by basic viral infections such as mycoplasma, parvovirus, HBV and $\mathrm{HCV}$, cytomegalovirus, varicella, toxoplasma and Ebstein-Barr virus were reported (3,9,15-18). While non-specific infections were detected in most of our patients, 1 had varicella, 1 had HBV, 1 had HEV and 1 had tuberculosis infection. Patients who were admitted with AIHA presentation must be questioned for previous infections and serological and microbiological studies must be performed on patients with an active infection in order to reveal a potential agent.

Neutrophil count was significantly lower in the patients diagnosed secondary AIHA than those in patients with primary AIHA. Total bilirubin level was higher in patients with primary AIHA than those in patients with secondary AIHA. When the patients with primary and secondary AIHA were compared regarding hemogram and biochemical parameters, no difference was found. High DAT positivity $(+++/++++)$ was higher in patients with primary AIHA than in patients with secondary AIHA. We considered that low neutrophil count in patients with secondary AIHA may be due to the underlying immunodeficiency and auto-inflammatory disease. DAT positivity and clinical findings suggest the presence of AIHA, however, it should be kept in mind that DAT may be positive in chronic liver diseases, malignancies, patients who frequently undergo transfusion due to drug use and in patients who have underwent bone morrow or organ transplantation. Moreover, DAT may also be positive in auto-inflammatory diseases (SLE, rheumatoid arthritis, etc.) and immunodeficiencies (autoimmune lymphoproliferative disease (ALPS), SCID, etc.) (19-26). DAT may be found as negative in approximately $3-11 \%$ of the patients with AIHA.20,25 Patients with negative DAT result, patients who underwent transfusion and patients who underwent a transplantation were excluded.

AIHA caused by warm antibodies is the most common type of AIHA seen in children and occurs with IgGtypeantibodies activated at room temperature or $37 \mathrm{oC}$. In cold AIHA, there are IgM-type antibodies typically binding to erythrocytes at temperatures between $0 \mathrm{oC}$ and $37 \mathrm{oC}$. Similar to the series in literature and as expected, the positivity rate of IgG-type antibodies was high. In patients with high positive results of DAT, low levels of $\mathrm{Hb}$ were found while need for transfusion was higher; the duration of the treatment was longer; response to the treatment lasted longer; and prognoses were worse. Moreover, DAT positivity was higher in patients with primary AIHA than in patients with secondary AIHA $(3,9,11,12)$.
AIHA may result in death. The therapeutic decisions should be made according to the growth rate and severity of anemia. The recommended treatments rely on patientbased individual experiences. Observation may be sufficient in a patient with moderate level of anemia due to a viral infection. The treatment should be initiated in patients with more severe anemia and in patients with rapid decrease in $\mathrm{Hb}$ level (1-4). If the patient has a serious intravascular hemolysis, renal blood flow and the amount urine should be carefully monitored. In children with warm antibody-related AIHA, the treatment should start with monitoring, erythrocyte transfusion and corticosteroid. Intravenous immunoglobulin, plasma exchange in chosen patients, and RTX as targeted treatment can be added to the treatment $(4,27)$. We tried to prefer corticosteroid treatments as first-line treatment in our patients diagnosed with AIHA. In cases (patients with active reactions, etc.) in which corticosteroid therapy could not be used, we preferred IVIG treatment as second option. In patients who were refractory to the treatment or who relapsed, we used other immunosuppressive treatments (MMF, cyclosporine, and RTX), splenectomy and recently plasma exchange. Cushing's syndrome, stria, hypertrichosis, and gastrointestinal side effects were observed secondary to corticosteroid treatment. Venous sinus thrombosis secondary to high-dose steroid treatment was found in one patient and IVIG treatment was carried on. In cyclosporine treatment, hirsutism, and high blood pressure were found. MMF had fewer side effects and its use was more readily than cyclosporine. Side effects occurring due to the drugs and insufficient treatments affected our treatment alterations. No complication was observed in patients who received RTX. In our study, one patient received 4 doses $(375 \mathrm{mg} /$ $\mathrm{m} 2)$ while another patient received 6 doses $(375 \mathrm{mg} / \mathrm{m} 2)$. Among 4 patients, one underwent 4 plasma exchange sessions whereas 3 underwent 3 plasma exchange sessions. When the literature is reviewed, high-dose steroid, IVIG, cyclosporine, MMF and splenectomy were performed in previous studies while RTX and plasmapheresis have become treatments preferred $(28,29)$. In patients who develop complications due to corticosteroid, RTX , cyclosporine and MMF, immunosuppressive drugs such as azathioprine and campath-1H and chemotherapeutics such as 6-merkaptopurin and cyclophosphamide can be used. Autologous stem cell transplantation may be performed in refractory AIHA (30).

Secondary AIHA is a condition that occurs secondary to inflammatory or autoimmune diseases such as Sjogren's syndrome, scleroderma, dermatomyositis, ulcerative colitis, Crohn'sdisease, and autoimmune thyroid; however it is most commonly seen in SLE (31). In the study from Mayo Clinic and in the largest pediatric study by Aladjiji et al., autoimmune/inflammatory diseases were the 
most commonly detected secondary diseases in patients diagnosed with AIHA $(11,12)$. Similarly, the most common causes of AIHA were autoimmune diseases in the study by Vaglio et al. (32) SLE, autoimmune hepatitis, antiphospholipid syndrome, primary sclerosing cholangitis, Evans' syndrome and rheumatoid arthritis were main autoimmune diseases detected. In the study by Aladjiji et al. (11), secondary AIHA incidence was $63 \%$ whereas it was $11 \%$ for autoimmune disorders. In our study, secondary AIHA was found in $58 \%$ of the patients. The most commonly detected causes of secondary AIHA were immunodeficienciesand autoimmune diseases. Evans syndrome and SLE were the most common autoimmune diseases. While some of our patients were followed up with autoimmune/auto-inflammatory diseases, immunodeficiency was diagnosed in their follow-ups.

In malignancies such as chronic myeloid, leukemia, lymphoma, multiple myeloma and myelodysplasia, AIHA may develop before or during the disease. In the study from Mayo Clinic, one patient had chronic myeloid leukemia but not lymphoma. Three patients had non-Hodgkin lymphoma in the study by La Sapienza and 8 patients had a malignancy (breast cancer, thyroid cancer, lymphoma, colon cancer and acute myeloid leukemia) in the study by Aladjiji et al. (11). In our study, Hodgkin's lymphoma developed in 3 patients. Two of the patients in whom Hodgkin's lymphoma was detected were observed to have been followed up with AIHA secondary to ALPS and 1 patient with Evans syndrome. It may also be seen with congenital immunodeficiencies accompanied by immune cytopenia. It is particularly seen in immunodeficiency syndrome that often changes and in Wiskott-Aldrich syndrome. AIHA may also be seen in acquired immunodeficiencies occurring after human immunodeficiency virusor organ transplantation (33). In our study, immunodeficiency was found in $19 \%$ of the patients diagnosed with AIHA and in about $43 \%$ of the patients diagnosed with secondary AIHA. PMG3 mutation, LRBA deficiency, RAG1 deficiency, ZAP70 deficiency and XLF-2 deficiency were primary immunodeficiencies that can be typed with the use of examinations of all genes-analysis. As a result of WES, LRBA deficiency was found in 2 patients followed up with AIHA secondary to ALPS and ZAP70 deficiency was found in one patient followed up with AIHA secondary to SLE. We had patients followed with immunodeficiency that could not be typed in the era where WES technology was unavailable. In the study by Aladjiji et al (11), humoral immunodeficiency was detected in 17 patients, non-identified primary immunodeficiency in 5, combined immunodeficiency in 2, adenosine deaminase deficiency in 2 and HLA class 2 deficiency in 1 patient.
Evans syndrome and ALPS that should be considered in the distinctive diagnosis of organomegaly, lymphadenopathy, and immunodeficiencies $(34,35)$. Evans syndrome was found in 99 patients in a French study, in 20 patients in the study of Junjie Fan et al. (36) and in 6 patients in the study of Naithani et al. $(11,37)$. In our study, Evans syndrome was found in 6 patients.

Although immune hemolytic anemia caused by drugs is not common in children. methyldopa, penicillin, cephalosporin, tetracycline, erythromycin, ribavirin, acetaminophen and ibuprofen may also be the cause of hemolytic anemia (38). Paroxysmal nocturnal hemoglobinuria $(\mathrm{PNH})$ which is rarely seen in children should be considered $(39,40)$. In our study, PNH was rarely seen in childhood, and no AIHA caused by drugs was observed.

In conclusion, AIHA may be either primary or secondary to underlying diseases such as autoimmune diseases, malignancies, infections and immunodeficiencies. In our study, the rate of patients with immunodeficiency was quite high. By advent of WES technology, we believe that primary immunodeficiencies may be the most common underlying diseases in patients especially with autoimmune-induced AIHA. Patients diagnosed with AIHA should be examined for immunodeficiencies.

\section{ETHICAL DECLARATIONS}

Ethics Committee Approval: The study was carried out with the permission of Erciyes University Faculty of Medicine Clinical Researchs Ethics Committee (Date: 16.06.2017, Decision No: 2017/348).

Informed Consent: Because the study was designed retrospectively, no written informed consent form was obtained from patients.

Referee Evaluation Process: Externally peer-reviewed.

Conflict of Interest Statement: The authors have no conflicts of interest to declare.

Financial Disclosure: The authors declared that this study has received no financial support.

Author Contributions: All of the authors declare that they have all participated in the design, execution, and analysis of the paper, and that they have approved the final version.

Acknowledgment: The authors thank Prof. Dr. Mehmet Akif Özdemir for their valuable contributions to the study.

\section{REFERENCES}

1. Liebman HA, WeitzIC. Autoimmune hemolytic anemia. Med Clin North Am 2017; 101: 351-9.

2. Aladjidi N, Jutand MA, Beaubois C, et al. Reliable assessment of the incidence of childhood autoimmune hemolytic anemia. Pediatr Blood Cancer 2017; 64: 12. 
3. Sarper N, Çakı Kılıç S, Zengin E, et al. Management of autoimmune hemolytic anemia in children and adolescents: A single center experience. Turk J Haematol 2011; 28: 198-205.

4. Vagace JM, Bajo R, Gervasini G. Diagnostic and therapeutic challenges of primary autoimmune haemolytic anaemia in children. Arch Dis Child 2014; 99: 668-73.

5. Carson JL, Grossman BJ, Kleinman S, et al. Clinical Transfusion Medicine Committee of the AABB. Red blood cell transfusion: a clinical practice guideline from the $\mathrm{AABB}^{*}$. Ann Intern Med. 2012; 157: 49-58.

6. Go RS, Winters JL, Kay NE. How I treat autoimmune hemolytic anemia. Blood.2017; 129: 2971-9.

7. Ladogana S, Maruzzi M, Samperi P, et al. Diagnosis and management of newly diagnosed childhood autoimmune haemolytic anaemia. Recommendations from the Red Cell Study Group of the Paediatric Haemato-Oncology Italian Association. Blood Transfus 2017;15: 259-67.

8. Ozcan A, Canpolat M, Doganay S, et al. Cerebral sinus venous thrombosis and prothrombotic risk factors in children: a singlecenter experience from Turkey. J Pediatr Hematol Oncol 2018; 40: 369-72.

9. Yaralı N, Bilir ÖA, Erdem AY, et al. Clinical features and treatment of primary autoimmune hemolytic anemia in childhood. Transfus Apher Sci 2018; 57: 665-8.

10.10.Naithani R, Agrawal N, Mahapatra M, et al. Autoimmune haemolytic anemia in children. Pediatr Hematol Oncol 2007; 24 309-15.

11.Aladjidi N, Leverger G, Leblanc T, et al. Centre de Référence National des Cytopénies Auto-immunes de l'Enfant (CEREVANCE). New insights into childhood autoimmune hemolytic anemia: a French national observational study of 265 children. Haematologica 2011; 96: 655-63.

12.Sankaran J, Rodriguez V, Jacob EK, et al. Autoimmune hemolytic anemia in children: mayo clinic experience. J Pediatr Hematol Oncol 2016; 38: 120-4.

13. Berentsen S, Sundic T. Red blood cell destruction in autoimmune hemolytic anemia: role of complement and potential new targets for therapy. Biomed Res Int 2015; 2015: 363278.

14. Hill QA, Hill A, Berentsen S. Defining autoimmune hemolytic anemia: a systematic review of the terminology used for diagnosis and treatment. Blood Adv 2019; 3: 1897-906.

15. Apak H, Karaman S, Doğan Y,etal. Varicella-induced haemolytic anemia with hepatitis. Ann Hematol 2006; 85: 64-5.

16.Zhang QL, Jia LJ, Zhang JB, et al. HBV and HCV coinfection associated with warm-type autoimmune hemolytic anemia: a case report. Turk J Haematol 2014; 3: 328-31.

17. Yen YF, Lan YC, Huang CT, et al. Human immunodeficiency virus infection increases the risk of incident autoimmune hemolytic anemia: a population-based cohort study in Taiwan. J Infect Dis 2017; 216: 1000-7.

18. Patiroglu T, Ozcan A, Karakukcu M, et al. Mycophenolate mofetil-induced pseudotumor cerebri in a boy with autoimmune lymphoproliferative disease. Childs Nerv Syst 2011; 27: 853-5.

19. Parker V, Tormey CA. The direct antiglobulin test: indications, interpretation, and pitfalls. Arch Pathol Lab Med 2017; 141: 30510.

20. Kim TO, Grimes AB, Kirk S, Arulselvanet al. Association of a positive direct antiglobulin test with chronic immune Thrombocytopenia and use of second line therapies in children: A multi-institutional review. Am J Hematol 2019; 94: 461-6.

21.Farmer JR, Foldvari Z, Ujhazi B, et al. Outcomes and treatment strategies for autoimmunity and hyperinflammation in patients with RAG deficiency.J Allergy Clin Immunol Pract 2019; 7: 197085.
22.Patiroglu T, Haluk Akar H, Gilmour K, et al. A case of XMEN syndrome presented with severe auto-immune disorders mimicking autoimmune lymphoproliferative disease. Clin Immunol 2015; 159: 58-62.

23. Patiroglu T, Gungor HE, Unal E. Autoimmune diseases detected in children with primary immunodeficiency diseases: results from a reference centre at middle anatolia. Acta Microbiol Immunol Hung 2012; 59: 343-53.

24. Patiroglu T, Akar HH, Gilmour K, et al. Atypical severe combined immunodeficiency caused by a novel homozygous mutation in Ragl gene in a girl who presented with pyoderma gangrenosum: a case report and literature review. J Clin Immunol 2014; 34: 792-5.

25. Miller J, Cai W, Andrews J, et al. A case series of pediatric patients with direct antiglobulin test negative autoimmune hemolytic anemia. Transfusion 2019; 59: 2528-1.

26.Patiroglu T, Klein C, Gungor HE, et al. Clinical features and genetic analysis of six patients with Wiskott-Aldrich syndrome reporting two novel mutations: experience of Erciyes University, Kayseri, Turkey. Genet Couns 2016; 27: 9-24.

27. Barros MM, Blajchman MA, Bordin JO. Warm autoimmune hemolytic anemia: recent progress in understanding the immunobiology and the treatment. Transfus Med Rev. 2010;24:195-210.

28. Dierickx D, Kentos A, Delannoy A. The role of rituximab in adults with warm antibody autoimmune haemolytic anemia.Blood 2015; 125: 3223-9.

29. von Baeyer H. Plasmapheresis in immune hematology: review of clinical outcome data with respect to evidence-based medicine and clinical experience. Ther Apher Dial 2003; 7: 127-40.

30.Barcellini W. Immune Hemolysis: Diagnosis and Treatment Recommendations. Semin Hematol 2015; 52: 304-12.

31. Hirano $\mathrm{Y}$, Itonaga $\mathrm{T}$, Yasudo $\mathrm{H}$, et al. Systemic lupus erythematosus presenting with mixed-type fulminant autoimmune hemolytic anemia. Pediatr Int 2016; 58: 527-30.

32. Vaglio S, Arista MC, Perrone MP, et al. Autoimmune haemolytic anemia in childhood: serologic features in 100 cases. Transfusion 2007; 47: 50-4.

33. Becheur M, Bouslama B, Slama H, et al. [Autoimmune haemolytic anemia in children]. Transfus Clin Biol 2015; 22: 291-8.

34. Miano M. How I manage Evans Syndrome and AIHA cases in children. Br J Haematol 2016; 172: 524-34.

35. Price V. Auto-immune lymphoproliferative disorder and other secondary immune thrombocytopenias in childhood. Pediatr Blood Cancer. 2013; 60: S12-4.

36. Fan J, He H, Zhao W, et al. Clinical features and treatment outcomes of childhood autoimmune hemolytic anemia: a retrospective analysis of 68 Cases. J Pediatr Hematol Oncol 2016; 38: $50-5$.

37. Naithani R, Agrawal N, Mahapatra M, et al. Autoimmune haemolytic anemia in India: clinico-hematological spectrum of 79 cases. Hematology 2006; 11: 73-6.

38. Garratty G. Immune hemolytic anemia caused by drugs. Expert Opin Drug Saf 2012; 11: 635- 42.

39. Albayrak M, Kaya Z, Kocak U, et al. Fatal autoimmune hemolytic anemia in a child with B-cell leukemia. Erciyes Med Journal 2009; 31: 37-40.

40.Parker CJ. Update on the diagnosis and management of paroxysmal nocturnal hemoglobinuria. Hematology Am Soc Hematol Educ Program 2016; 2016: 208-16. 